\title{
Utilisation des matériels d'irrigation par aspersion. Diagnostic de fonctionnement au champ
}

\author{
Jean Dubalen \\ Ingénieur à TRAME-BCMA
}

L'irrigation par aspersion occupe, en France, environ $85 \%$ de la surface agricole annuellement arrosée.

Trois systèmes principaux sont utilisés : l'Enrouleur, sur 900000 ha environ; la Couverture intégrale en petits arroseurs, de l'ordre de 100000 ha; le système Pivot, de l'ordre de 100000 ha.

L'irrigant choisit l'un ou l'autre de ces systèmes en fonction de ses disponibilités financières, de ses disponibilités en main-d'œuvre, et d'un certain nombre de contraintes techniques (cultures arrosées, configuration du parcellaire, type de sol, climat, etc.).

Pour qu'une installation de l'irrigation soit bien adaptée, il faut qu'il y ait une bonne corrélation entre les performances possibles de l'installation caractérisées par le débit d'équipement, les besoins de la sole arrosée, et les disponibilités de la ressource en eau.

En outre, pour que l'irrigant rentabilise au mieux les investissements engagés, il convient que le matériel d'arrosage soit correctement utilisé, les critères de bonne utilisation pouvant se résumer comme suit :

- Apporter au moment opportun et avec une précision suffisante la quantité d'eau nécessaire.

- Répartir cette dose d'eau de façon suffisamment homogène sur le parcellaire.

- Assurer un arrosage de la meilleure qualité possible : pluie suffisamment fine et d'intensité acceptable par le sol et la culture.

Les bons irrigants ont eu conscience :

- qu'il n'était pas toujours facile d'obtenir une précision et une qualité d'irrigation satisfaisantes avec les équipements mis a leur disposition par les constructeurs ;

- que le "manque à gagner " consécutif à une pratique trop approximative de l'irrigation était loin d'être négligeable.

Aussi, pour permettre aux irrigants qui le souhaitaient d'utiliser au mieux leur équipement d'arrosage, la CRAMP (Chambre Régionale d'Agriculture Midi-Pyrénées) et le BCMA (Bureau de Coordination du Machinisme Agricole) ont lancé, en 1988, l'opération "Diagnostic au champ du fonctionnement des équipements d'irrigation 》.

De 1988 à 1991, les conseillers irrigation de la région Midi-Pyrénées ont "diagnostiqué » :

- 382 installations d'irrigation par Enrouleur :
- 78 installations d'irrigation par Couverture intégrale en petits arroseurs ;

- 8 installations d'irrigation par Pivot.

Le RNED-HA s'est intéressé à l'opération afin qu'elle se généralise aux autres régions concernées par l'irrigation.

\section{Conduite d'un diagnostic}

L'opération " Diagnostic " a pour but de permettre aux irrigants qui le souhaitent de mettre en évidence les faiblesses constatées dans l'utilisation de leur équipement d'arrosage, d'apporter les correctifs nécessaires à une bonne utilisation de leur installation afin d'optimiser son intérêt économique, et d'économiser l'eau.

Le diagnostic d'une installation d'arrosage se déroule en 4 phases :

$I-Y$ a-t-il une bonne corrélation entre :

- Les besoins de la sole irriguée.

- Les possibilités du matériel d'arrosage.

- Les disponibilités de la ressource en eau.

2 - L'irrigant connaît-il bien son installation d'arrosage?

- Débit du (ou des) arroseur(s)?

- Pression de fonctionnement?

- Dose annoncée?

- Hétérogénéité d'apport constatée ?, etc.

3 - Contrôle de l'installation en fonctionnement :

- Vérification des manomètres.

- Contrôle du busage.

- Mesure des pressions de fonctionnement des arroseurs.

- Evaluation du débit du ou des arroseurs.

- Calcul de la dose moyenne apportée.

- Variations de la dose apportée.

- Réglage des arroseurs.

- Homogénéité de répartition par pluviomètres (sur pivot uniquement).

4 - Remise à l'irrigant des résultats du diagnostic:

- Comparaison des chiffres (débit, pression, dose, etc.) :

- annoncés par l'irrigant,

- contrôlés,

- souhaitables.

- Modifications à apporter à l'installation d'arrosage ou à la façon de l'utiliser. 
Les enseignements de l'opération Diagnostic

\section{Irrigation par enrouleur}

Le dépouillement des 382 fiches diagnostic relatives aux installations d'arrosage par enrouleur a permis de dresser le constat suivant :

Adaptation du débit des installations aux besoins de la sole arrosée : le débit est conforme aux besoins dans seulement $30 \%$ des cas. Il est inférieur dans $39 \%$ des cas. Il est supérieur dans $31 \%$ des cas.

Les raisons principales d'un équipement mal adapté sont le plus souvent :

- Le manque de rigueur de l'étude préalable visant à définir les caractéristiques de l'équipement nécessaire ;

- l'exagération de la surface affectée à l'installation en regard des performances de celle-ci et des risques climatiques locaux.

Précision des apports d'eau: $\mathrm{Si}$ on compare les doses annoncées par les irrigants et les doses contrôlées par les conseillers, on s'aperçoit (cf. tableau) que le nombre des irrigants qui apportent avec une précision acceptable les doses qu'ils annoncent, n'excède pas $24 \%$.

Ce constat est particulièrement préoccupant. Les raisons essentielles paraissent être :

- la méconnaissance trop fréquente du débit des canons ; - le contrôle des apports avec seulement un (parfois 2) pluviomètre.

Homogénéité de répartition de l'eau: Parmi les causes d'une homogénéité de répartition insuffisante, souvent constatée, on peut noter :

- des intervalles de passage trop importants, compte tenu de l'importance du vent dans la région, l'irrigant cherchant à limiter le nombre de déplacements de l'enrouleur ;

- le mauvais réglage du canon travaillant trop souvent en secteur dissymétrique, particulièrement sur les parcelles en pente ;

- le mauvais fonctionnement des systèmes mécaniques de régulation d'avancement: A peine un quart d'entre eux fonctionnent correctement.

Qualité de l'arrosage : La pression de fonctionnement du canon constitue le critère d'évaluation le plus commode. Or, $69 \%$ des enrouleurs diagnostiqués étaient dépourvus de manomètre au canon, ce qui interdit tout contrôle rapide et précis de la part de l'irrigant.

Les conseillers ont observé un nombre important de canons fonctionnant :

— à pression trop faible : pluie d'intensité trop élevée ;

— ou parfois trop forte: plus grande sensibilité au vent.

\section{Irrigation par Couverture intégrale en petits arroseurs}

Soixante-dix-huit installations de ce type ont été "diagnostiquées ", le constat est le suivant :

Adaptation du débit des installations aux besoins de la sole arrosée: Il s'agit ici d'installations fixes occupant une surface déterminée. Le débit de ces installations est, en général, en bonne corrélation avec les besoins de la sole arrosée, dans la mesure où les irrigants n'ont pas augmenté par la suite le nombre d'arroseurs prévus dans le projet initial.

Précision des apports d'eau: La comparaison des doses annoncées et des doses contrôlées fait apparaître que le nombre d'irrigants qui apportent avec une précision acceptable les doses qu'ils annoncent, atteint ici $41 \%$. La précision des apports est donc meilleure en couverture intégrale qu'en enrouleur.

Lorsque les doses sont mal maîtrisées en couverture intégrale, les raisons paraissent être :

- le contrôle de l'apport avec seulement 1 (parfois 2) pluviomètre : ceci conduit à une évaluation fausse du fait d'une certaine hétérogénéité de répartition de l'eau sous les arroseurs, inévitable même sur les installations bien conçues ;

- la connaissance trop approximative du débit des arroseurs, beaucoup d'irrigants ne possédant pas les abaques de ceux-ci ;

- le temps des positions d'arrosage, lorsque l'irrigant le choisit, davantage par commodité qu'en référence à la pluviométrie horaire de l'installation.

Homogénéité de répartition sur le parcellaire : L'homogénéité de répartition est souvent insuffisante pour deux raisons essentielles :

- pour limiter les investissements, les implantations adoptées sont souvent trop lâches en regard de l'importance du vent dans la région, réduisant la portée pratique des arroseurs ;

- l'écart de pression constaté entre arroseurs extrêmes sur le parcellaire est souvent trop important : les irrigants ne possédant pas, en général, de moyen de contrôle des pressions de fonctionnement des arroseurs, se contentent le plus souvent d'une comparaison visuelle des jets.

Qualité de l'arrosage : En couverture intégrale, l'intensité de pluie (pluviométrie horaire) et la finesse des gouttes, sont acceptables dans la plupart des situations. Les pressions moyennes relevées par les conseillers sont, le plus souvent, correctes : dans $20 \%$ des cas seulement, on constate des pressions moyennes aux arroseurs inférieures à 3,5 bars.

\section{Irrigation par pivot}

Le diagnostic d'une installation d'arrosage par enrouleur ou par couverture intégrale demande 2 ou 3 heures. Le diagnostic d'une installation d'arrosage par pivot demande au moins une journée.

De ce fait, 8 pivots seulement ont été "diagnostiqués 》 au cours de la période considérée. Il a malgré tout été possible d'en tirer quelques enseignements.

Adaptation du débit de l'installation aux besoins de la sole arrosée: Ce système d'arrosage, difficilement extensible, évolue sur une surface à arroser bien déterminée : le débit de l'appareil est généralement bien adapté aux besoins. 
REGION MIDI-PYRENEES

\section{DIAGNOSTIC AU CHAMP DES INSTALLATIONS \\ D'IRRIGATION \\ 1988 - 1991}

\begin{tabular}{|c|c|c|}
\hline \multirow[b]{2}{*}{ Diagnostics réalisés } & Enrouleurs & \multirow{2}{*}{$\begin{array}{r}\begin{array}{c}\text { Couverture } \\
\text { Intégrale }\end{array} \\
78\end{array}$} \\
\hline & 382 & \\
\hline $\begin{array}{l}\text { I - PRECISION DE LA DOSE APPORT } \\
\text { Ecart entre la dose annoncée et la dose } \\
\text { contrôlée } \\
\text {. Inférieur à } 10 \% \\
\text { Entre } 10 \text { et } 20 \% \\
\text {. Supérieur à } 20 \%\end{array}$ & $\begin{array}{l}24 \% \\
30 \% \\
46 \%\end{array}$ & $\begin{array}{l}41 \% \\
25 \% \\
34 \%\end{array}$ \\
\hline $\begin{array}{l}\text { II - HOMOGENEITE DE REPARTITIO } \\
\text { ENROULEUR } \\
\text { Espacement trop important entre } \\
\text { passages } \\
\text {. Secteur canon dissymétrique } \\
\begin{array}{l}\text { Variation de la } \\
\text { vitesse } \\
\text { d'avancement } \quad\left\{\begin{array}{l}\text { inférieure à } 10 \% \\
\text { entre } 10 \text { et } 20 \% \\
\text { supérieure à } 20 \%\end{array}\right.\end{array}\end{array}$ & $\begin{array}{l}65 \% \\
59 \% \\
24 \% \\
37 \% \\
39 \%\end{array}$ & \\
\hline $\begin{array}{l}\text { COUVERTURE INTEGRALE } \\
\text { Implantation trop lâche } \\
\text { Ecart de pression entre arroseurs } \\
\text { extrêmes supérieur à } 20 \%\end{array}$ & & $\begin{array}{l}70 \% \\
56 \%\end{array}$ \\
\hline $\begin{array}{l}\text { III - QUALITE DE L'ARROSAGE } \\
\text { PRESSION MOYENNE AUX ARROSEURS } \\
\text {. Convenable } \\
\text { Trop faible } \\
\text {. Trop Forte }\end{array}$ & $\begin{array}{l}52 \% \\
38 \% \\
10 \%\end{array}$ & $\begin{array}{l}58 \% \\
20 \% \\
22 \%\end{array}$ \\
\hline
\end{tabular}

A noter que, si la ressource en eau n'est pas limitée, un léger excédent de débit, par rapport aux besoins, ne pénalise pas lourdement les investissements.

Précision des apports d'eau: $\mathrm{Si}$ le débit du pivot a été correctement calculé par rapport à la surface arrosée, et si l'appareil fonctionne de façon régulière, l'apport mensuel nécessaire peut être respecté avec une précision suffisante.

On observe, toutefois, que les doses unitaires moyennes contrôlées ne correspondent avec précision aux doses annoncées, que dans la moitié des installations « diagnostiquées" (voir exemple).

Compte tenu des remarques précédentes, la précision de la dose a moins d'importance dans le cas du pivot que dans le cas des autres systèmes d'arrosage, sauf si le pivot arrose plusieurs cultures de besoins différents.

Homogénéité de répartition: C'est la phase la plus importante et la plus longue du diagnostic. Le contrôle de la répartition ne peut être réalisé qu'au moyen de pluviomètres ou de récipients adéquats disposés tous les 5 mètres selon un rayon arrosé par le pivot ; 2 contrôles successifs sont généralement nécessaires.

Les relevés des pluviomètres permettent de dresser un diagramme pluviométrique à partir duquel le conseiller juge la qualité de la répartition de l'eau distribuée par l'appareil.

Si des zones de surdosage ou de sous-dosage apparaissent sur le diagramme, l'irrigant doit contrôler, dans ces 


\section{DIAGNOSTIC DU FONCTIONNEMENT D'UN PIVOT AU CHAMP DIAGRAMME PLUVIOMÉTRIQUE}

Schéma descriptif du pivot et courbe pluviométrique en fonction de la surface
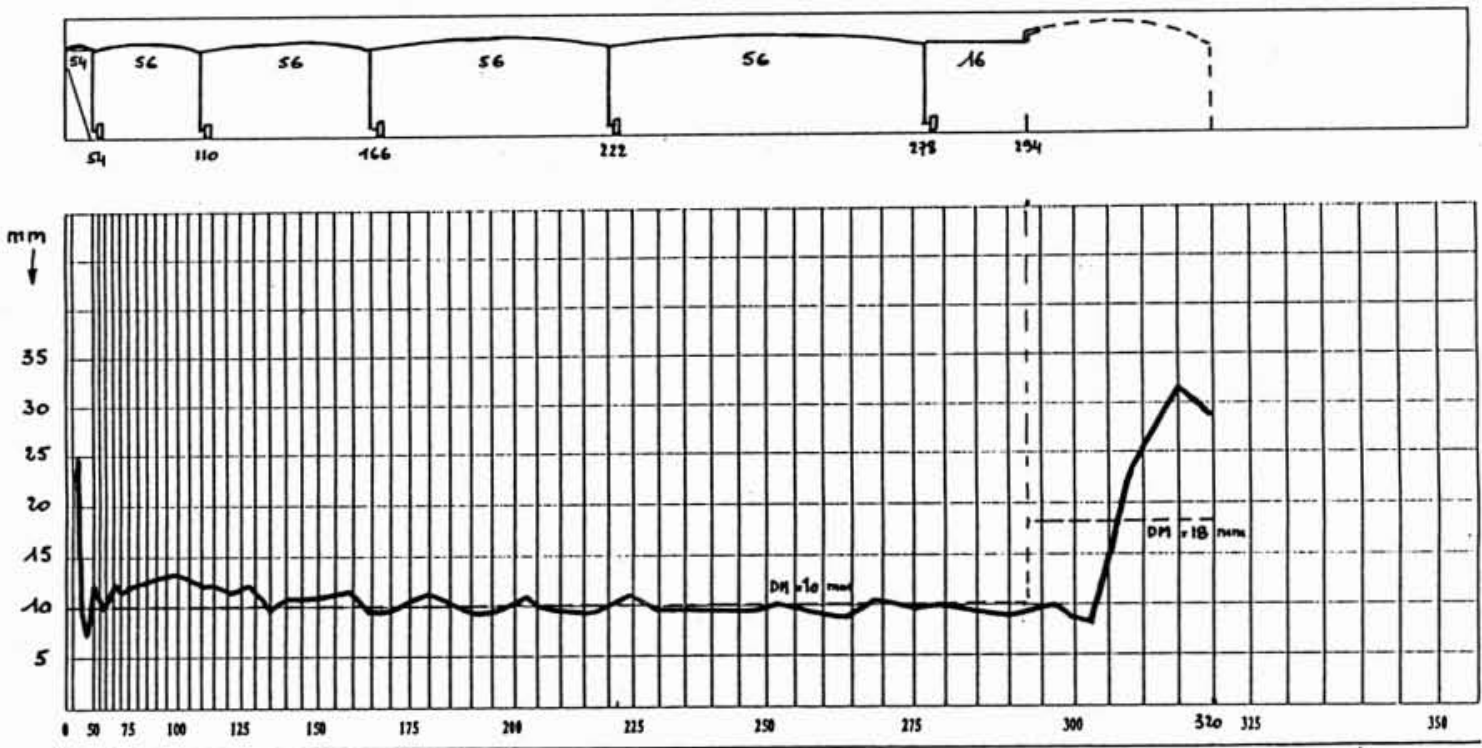

Dose annoncée : 13 à $14 \mathrm{~mm}$

Dose MOYENNE calculée : $10 \mathrm{~mm}$ (18 $\mathrm{mm}$ sous canon)

Répartition HOMOGÈNE sauf sous canon d'extrémité

Réglage du canon d'extrémité à revoir
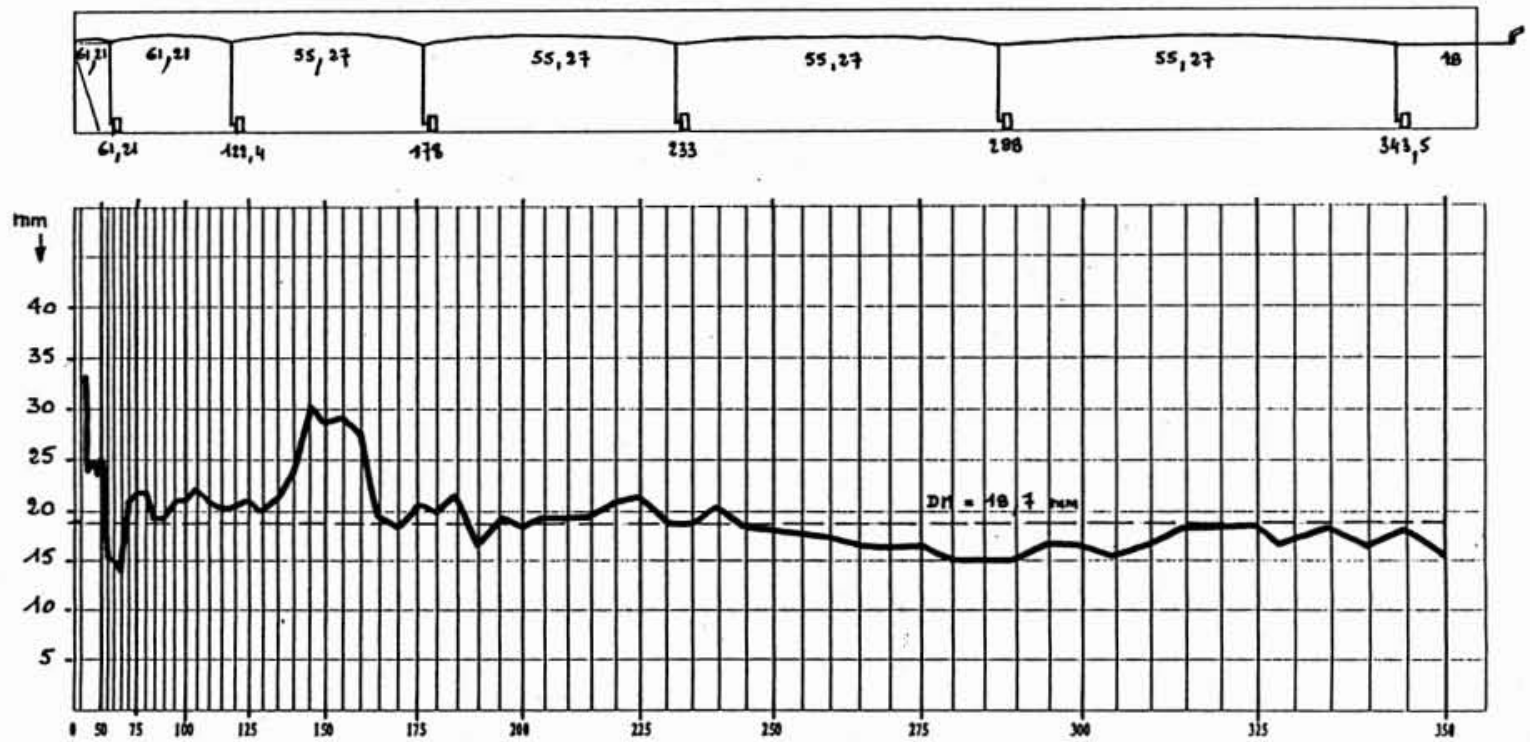

- Dose annoncée : $18 \mathrm{~mm} \quad$ Dose MOYENNE calculée : $18,7 \mathrm{~mm}$

- Homogénéité de répartition MÉDIOCRE

- Vérifier BUSAGE par rapport au LISTING au niveau des travées 2 et 3

- Canon d'extrémité non contrôlé : Fonctionne uniquement dans les angles 
zones, le busage de son pivot par rapport au listing de busage établi par le constructeur : une erreur au montage a pu être commise. Si ce n'est pas le cas, il convient de faire recalculer le busage du pivot.

Le diagramme fait apparaittre aussi les défauts éventuels de réglage du canon d'extrémité : busage inadapté, secteur d'évolution à modifier.

A noter que le surdosage constaté au niveau de la première travée (la plus proche de l'axe pivot) est presque toujours inévitable.

Qualité de l'arrosage: Le diagnostic comporte un contrôle des pressions de fonctionnement du pivot. Ce contrôle est généralement limité :

- à la pression " entrée pivot "; elle doit correspondre à la pression préconisée dans le listing de busage ;

- à la pression au niveau de la dernière tour, permettant de constater un éventuel encombrement du tube d'alimentation du pivot.
Evaluation du « manque à gagner » résultant de réglages insuffisamment précis

De nombreux irrigants semblent ne pas être sensibilisés à la nécessité de devoir apporter le plus grand soin à l'utilisation et aux réglages de leur équipement d'irrigation. Il convient donc qu'ils soient informés du "manque à gagner " résultant de mauvais réglages.

Des simulations, réalisées à partir de la fonction de production connue du maïs (logiciel LORA) ont permis d'évaluer ce " manque à gagner » traduit en perte de rendement. Cette perte est variable avec le degré d'imprécision des réglages pratiqués, mais elle peut atteindre, lorsque les imprécisions des différents réglages sont importantes et se cumulent :

- 20 à $25 \%$ du rendement en couverture intégrale;

- 30 à $35 \%$ du rendement dans le cas de l'enrouleur.

De tous les facteurs induisant cette perte, la précision des quantités d'eau apportées constitue le plus important.

\section{ÉVALUATION DU "MANQUE À GAGNER " CONSÉCUTIF DES RÉGLAGES OU À UNE UTILISATION TROP IMPRÉCIS DE L'INSTALLATION D'ARROSAGE}
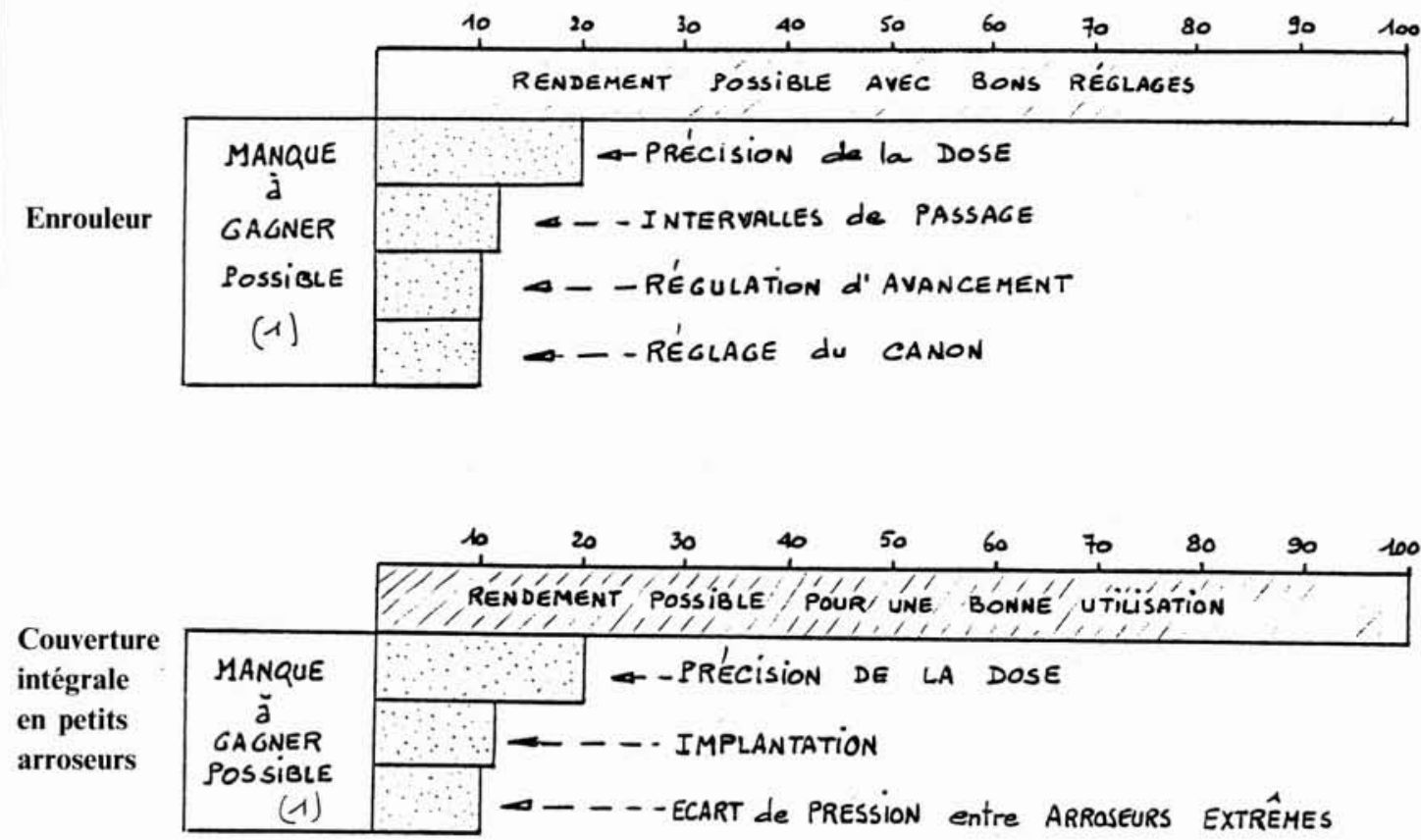

REMARQUE: les MANQUES À GAGNER résultant de DIFFÉRENTS MAUVAIS RÉGLAGES ou MAUVAISES UTILISATIONS PEUVENT SE CUMULER.

(1) Pour une MARGE D'ERREUR n'excédant pas $30 \%$. 


\section{Conclusions et perspectives}

Les résultats de l'opération "diagnostic au champ des équipements d'irrigation " conduite depuis 1988 en région MidiPyrénées, prouvent que de nombreux irrigants doivent s'efforcer de mieux maîtriser l'utilisation de leur équipement d'arrosage.

L'enjeu est important car :

- le manque à gagner consécutif à des réglages trop imprécis a pour conséquence une mauvaise rentabilité des investissements ;

- on peut difficilement parler de « conduite raisonnée de l'irrigation ", alors que les quantités d'eau apportées par l'installation ne sont pas connues de l'irrigant avec une précision suffisante.

Apporter des doses précises et homogènes sur l'ensemble du parcellaire, constitue la première condition indispensable pour conduire rationnellement l'irrigation et pour la rentabiliser.

\section{Arrosage par enrouleur}

L'opération "diagnostic " a montré que les systèmes mécaniques de régulation n'autorisent pas, en pratique, et sauf exception, une précision de réglage suffisante pour atteindre l'objectif que nous venons de définir.

Par. contre, les observations de terrain réalisées ces dernières années sur des enrouleurs équipés de systèmes électroniques de régulation, nous ont permis de constater que :

- ces systèmes permettent d'obtenir une très bonne régularité d'avancement : les variations de vitesse observées sur un enroulement complet excèdent rarement $5 \%$;

- la dose apportée est généralement beaucoup mieux maîtrisée par les irrigants lorsque leur enrouleur est équipé de ce type de régulation.

La majorité des régulations électroniques actuelles sont de simples régulateurs de vitesse. Il appartient dans ce cas à l'irrigant de déterminer la vitesse $(\mathrm{V})$ à laquelle le canon doit avancer pour appliquer la dose choisie (D). Il doit pour cela appliquer la relation simple, maintenant bien connue :

$$
\mathrm{V} \text { (mètres/heure) }=1000 \frac{\mathrm{Q}(\mathrm{m} 3 / \text { heure })}{\mathrm{D}(\mathrm{mm}) \times \mathrm{E}(\mathrm{m})}
$$

$Q$ étant le débit du canon (abaques) et $\mathrm{E}$ l'intervalle de passages de l'enrouleur.

Sur certains systèmes de régulation électronique plus sophistiqués (Irridoseur d'Irrifrance, Dosidis d'Irrimec, Raincontrol de Perrot, etc.), l'irrigant affiche directement la dose choisie, et divers paramètres permettant à l'appareil de calculer la vitesse correspondante.
La régulation électronique permet des réglages plus précis et plus faciles. L'irrigant doit, par ailleurs, apporter le plus grand soin :

- au choix des intervalles de passage adoptés, en fonction de l'importance locale du vent en période d'irrigation; - aux réglages du canon.

\section{Arrosage par Couverture Intégrale en petits arroseurs}

Le constat est moins sévère: les irrigants arrosent généralement mieux avec ce système qu'avec les enrouleurs, ce qui ne veut pas dire qu'ils ont intérêt à préférer la couverture intégrale, chacun des deux systèmes ayant son propre champ d'application.

On doit par ailleurs remarquer qu'un bon nombre d'utilisateurs de couverture intégrale aurait intérêt à utiliser ce système d'arrosage de façon plus rigoureuse, en :

- adoptant des implantations d'arroseurs plus resserrées en conditions ventées;

- harmonisant de façon plus précise, en fonction de la dose choisie, le temps des positions d'arrosage et la pluviométrie horaire de l'installation;

- améliorant la distribution de l'eau, de façon que l'écart de pression entre arroseurs extrêmes, sur le parcellaire, ne soit pas trop important.

\section{Arrosage par Pivot}

Le pivot apparaît actuellement comme l'un des systèmes d'arrosage par aspersion permettant le plus facilement de pratiquer une irrigation précise et de qualité, à condition : - que le busage ait été correctement calculé et respecté lors du montage de l'appareil ;

- que le canon d'extrémité soit correctement réglé.

Malgré ses avantages, l'extension de ce système restera pourtant limitée, en raison de sa conception même et du fait qu'il ne peut s'adapter qu'à certains parcellaires et à certaines soles arrosées.

Il est d'ailleurs peu probable, dans l'avenir, que l'un ou l'autre des systèmes d'arrosage par aspersion, prenne le pas sur les autres, chacun ayant son propre domaine d'utilisation.

Le constat que nous avons dressé prouve tout l'intérêt de poursuivre et de généraliser l'opération "diagnostic au champ ", avec un préalable indispensable: sensibiliser les irrigants à cette nécessité.

Ceci est particulièrement vrai pour les utilisateurs d'enrouleur qui demeure le système d'arrosage par aspersion le plus contraignant quant à l'importance des réglages qu'il nécessite.

Mais l'enrouleur restera, semble-t-il, pendant de longues années encore et en raison de sa grande souplesse d'utilisation, l'appareil d'arrosage le plus utilisé. 